\title{
3D Simulations of Block Caving Flow Using ESyS-Particle
}

\author{
W.R. Hancock The University of Queensland, Australia \\ D.K. Weatherley The University of Queensland, Australia
}

\begin{abstract}
The ESyS-Particle discrete element implementation is examined for validity in studying block caving flows through comparison against previous numerical studies. Unbonded granular assemblies are studied using triaxial simulations as a way to quantitatively characterise macroscopic friction angles with ranging size distributions and particle properties such as stiffness and per particle microscopic frictional coefficient. These results are used as a basis for $3 D$ quarter symmetry simulations of block caving flows. Calibration and comparisons are made against previous studies with ESyS-Particle exhibiting promising behaviour for the modelling of block caving flows. Ongoing studies will examine facets of flow such as fines migration and interactive draw in order to develop constitutive relationships and identify flow mechanisms.
\end{abstract}

\section{Introduction}

Block caving is an underground mining method used to extract ore from large low grade orebodies. By exploiting gravity, once an initial cavity is created below the orebody, it is then allowed to collapse under its own weight and the resulting fragmented rock is drawn from funnel shaped draw bells beneath. This process is used to mine large areas of underground ore with particular success, being one of the cheapest mining methods available (Julin, 1992). Due to the large initial capital required for block caving operations, understanding the issues of grade dilution, fines migration, particle size effects and the like pose a significant benefit to industry. By using the discrete element method (DEM) to represent each rock fragment as a particle, rock size distributions and model geometries can be recreated as necessary, allowing flow characteristics to be understood. An examination of flow widths with respect to these characteristics is undertaken, allowing comparison and calibration of the model against both physical and numerical studies. Results show significant promise in the area of flow analysis.

The ESyS-Particle code developed at the Earth Systems Science Computational Centre (ESSCC) has been used in previous years to study many areas that require vast simulations of particulate matter under interaction from external forces (Mora and Place, 1994; Abe et al., 2004). The very nature of this type of rock flow lends itself well to the DEM implemented in the ESyS-Particle code (Wang et al., 2006; Wang and Mora, 2007). The DEM models each rock fragment as a separate entity in the system, allowing the accurate tracking of particulate matter that would be infeasible in any physical modelling situation. Each rock fragment is also allowed a free range of movement producing results that are favourable over stochastic and various predetermined flow models. With regards to block caving, in order to produce realistic results, a large number of particles is required to be simulated. The parallel nature of ESyS-Particle and access to the Altix Supercomputer at UQ (Latham et al., 2004), lends itself favourably in regards to this limitation.

The following is an examination of the feasibility of ESyS-Particle with respect to block caving applications. Section 2 gives an overview of the DEM and methods used to calibrate and verify ESyS-Particle. Section 3 follows with a discussion of the results produced and Section 4 concludes with a short summary of work conducted and future directions. 


\section{Methodology}

The DEM (Cundall and Strack, 1979) is a widely used numerical technique, particularly for solving problems involving flow of granular assemblies or fragmentation. Unlike continuum-based methods, the DEM represents physical media as assemblies of discrete individual elements (often called particles) that interact with one another and other objects (such as elastic external walls) via simplified contact force laws. The simplest such law applies restoring forces to overlapping particles, with the magnitude of the restoring force proportional to the amount of overlap (the constant of proportionality is a Hookean elastic stiffness coefficient). By computing the net force acting on a given particle and applying Newton's Second Law, acceleration is obtained which is integrated to update the velocity and position of each particle. Timeintegration schemes vary but all aim to extrapolate velocities and positions forward in time by a small amount $\Delta \mathrm{t}$. A smaller value for $\Delta \mathrm{t}$ ensures greater accuracy of the solution, but at the expense of longer computation time. Likewise, increasing the number of particles more accurately reproduces physical phenomena, at considerable computational expense.

Whilst computational is expensive, the DEM has distinct advantages for modelling certain types of phenomena, most notably granular flow, the object of this paper. To model granular flow, the simplest forcedisplacement contact laws are insufficient. Consequently, for this study we utilise spherical particles with six degrees of freedom (i.e. both translational and rotational motion). Particle-pairs that come into contact experience elastic repulsion (the strength of which is determined by the microscopic elastic stiffness $\mathrm{K}_{\mathrm{N}}$ ) and frictional forces (with a specified coefficient of friction $\mu$ and shear stiffness $\mathrm{K}_{\mathrm{S}}$ ). The contact forces are partitioned into translational and rotational moments acting on the particles. Time-integration using these moments permits update of positions, orientations, angular and translational velocities of the particles. Elastic walls (with stiffness $\mathrm{K}_{\mathrm{W}}$ ) provide a mechanism to apply external forces (or boundary conditions) to an assembly of particles. By utilising a triangular mesh to represent walls, external boundaries of relatively arbitrary topology are achievable. Body forces such as gravity, are easily incorporated in DEMs by simply adding the equivalent body force into the calculation of net force for each particle.

The DEM implemented in the ESyS-Particle software utilised for this study includes rolling resistance designed to mimic the physics of spheres with a microscopically rough surface, rather than that of ideal, smooth spheres (Latham et al., 2005). The aim of such an implementation is to simulate the flow of rough granular materials utilising a minimal number of particles. Other DEMs often join partially overlapping spheres together into unbreakable clumps, the surficial roughness of which provides geometrical rolling resistance. Later in this paper, we examine the variation in macroscopic friction angles as the microscopic friction coefficients of ESyS-Particle spheres and PFC3D clumps are varied.

In order to calibrate the model, we focus not only on the model's ability to match caving flow characteristics but on a fundamental level aimed at highlighting both strengths and weaknesses of the code that may present problems during future simulations. Simulations using 2D flow are used as a stepping stone into the 3D setups; these tests encompass model parameters such as particle stiffness, friction coefficients, the effect of particle rotation and model geometry. Although they are not presented here, these tests produced results which have motivated the use of triaxial compression simulations aimed at understanding bulk friction angles produced by unbonded test samples. This information can then be compared to previous numerical studies and applied to flow models allowing a better understanding of model parameters on flow.

3D flow modelling setups have been designed through careful consideration of previous flow studies including that of the physical model used previously at the Julius Kruttschnitt Mineral Research Centre (JKMRC) (Power, 2004; Castro, 2006) and those completed numerically using PFC3D (Pierce et al., 2003). A number of setups have been used with varying geometries, boundary conditions and symmetry, the quarter symmetry model presented in this paper aims to mimic a setup that has been used previously in the aforementioned PFC3D numerical study. Calibration from these results is done through analysis of maximum flow width to height ratios using both the region of extracted particles and the region of particles that have gained mobility during the course of flow. 


\section{Results}

\subsection{Triaxial testing}

With one of the primary factors contributing to flow being the frictional interactions between particles, a number of triaxial test simulations were run to gain insight into the response of ESyS-Particle spheres with comparison to PFC3D clumps that have been used in previous numerical studies (Lorig and Cundall, 2000). The aim of the triaxial tests was to examine the hypothesis that the different rotational dynamics, implemented within the ESyS-Particle code, might provide a way of modelling rock without going to the extent of clumps, saving computational time and offering the possibility to model larger simulations. This has proven to be somewhat true; particles within the ESyS-Particle code are shown to exhibit a higher macroscopic frictional behaviour; however control of this macroscopic friction by parameter tuning is more difficult than for PFC3D clumps.

\subsubsection{Test setup description}

Triaxial tests are done over a prism of unbonded particles in 3D using a confining box where vertical walls are given a constant confining pressure and an unvarying strain rate is applied from the top and bottom walls. Peak measurements of stress observed on the top and bottom walls are then taken to calculate respective bulk friction angles. This testing procedure was then repeated multiple times over a number of samples with varying properties.

Two simulation setups were considered, one similar to the PFC3D case study and one using particles that are currently used in the quarter symmetry flow simulations of ESyS-Particle. Microscopic friction angle and particle distribution were the main variants within the simulations with some tests repeated at higher confining pressure to verify against the PFC3D results. In the following descriptions, a number of differences are highlighted between the two models and are explained as they arise.

Particle packing within ESyS-Particle is randomly distributed over a specified range. There is little control, using the current version of the code, over factors such as porosity or the particle size-distribution. This can lead to a packing with inevitably high porosity. In an attempt to reduce this, a greater range of smaller particles have been introduced within the sample, with the bulk of testing being done using particles 0.15 to $0.45 \mathrm{~m}$ in radius, porosity levels have been achieved in the order of 37\%. This is in comparison to the PFC3D samples which use an average clump radius of $0.35 \mathrm{~m}$ packed to similar porosity levels.

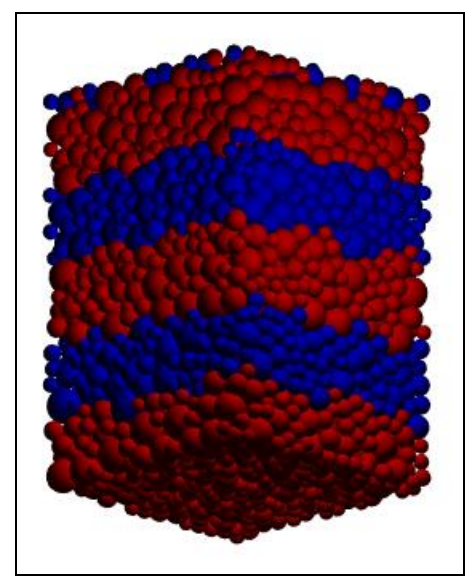

\section{Figure 1 Triaxial test initial packing}

The model measures $7 \times 7 \times 10 \mathrm{~m}$ (length $\times$ width $\times$ height) and is packed to contain approximately 4700 particles. Sidewall confinement pressure is then raised to the desired testing level and the top and bottom walls compress the sample at constant speed. A summary of model parameters is presented in Table 1 . 
Table 1 Triaxial testing configuration parameters

\begin{tabular}{ll}
\hline Parameter & Value \\
\hline Mean radius & $0.3 \mathrm{~m}$ \\
Particle density & $3333 \mathrm{~kg} / \mathrm{m}^{3}$ \\
Particle stiffness & $0.5 \mathrm{GPa} \times \mathrm{R}_{\text {mean }}$ \\
Particle normal/particle shear stiffness & 1.0 \\
Particle static friction coefficient $\mu$ & $0.1-1.0$ \\
Particle dynamic friction coefficient $\mu$ & $0.1-1.0$ \\
Viscosity & 0.1 \\
Confining pressure $\sigma$ & $0.5 \mathrm{MPa}$ for the majority of tests
\end{tabular}

Simulations were run to approximately $50 \%$ total strain and the peak of the stress-strain curve was taken as a method to calculate bulk friction angles similar to the method used within the PFC3D tests.

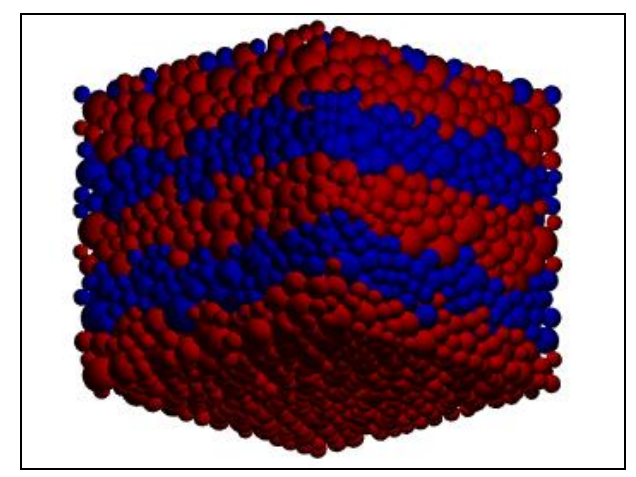

\section{Figure 2 Triaxial test final sample state}

The stress-strain response of the tests was compared to physical sand model testing and found to be reasonable in this regard. The stress versus strain curve for a typical simulation is shown in Figure 3.

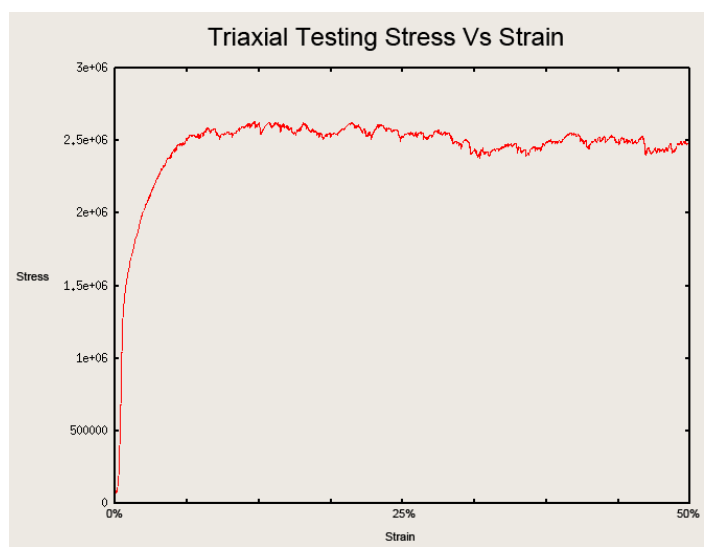

Figure 3 Typical stress versus strain response of ESyS-Particle 


\subsubsection{Macroscopic friction results and analysis}

The triaxial tests with ESyS-Particle demonstrate that macroscopic friction can be significantly higher than that of PFC3D, providing validation for the use of rotational particles within the flow simulations. Figure 4 shows the relationship between macroscopic friction angle and inter-particle coefficient of friction $(\mu)$ for both ESyS-Particle and PFC3D. Control of macroscopic friction is restricted to a narrow range $0.1<\mu<0.5$ and there is almost no variation in macroscopic friction angles for $\mu>0.5$. This is in contrast to the PFC3D results which show good response up to coefficients of 2 . The shape of the particles (clumps versus spheres) and the different implementations of rotational dynamics are thought to cause these differences.

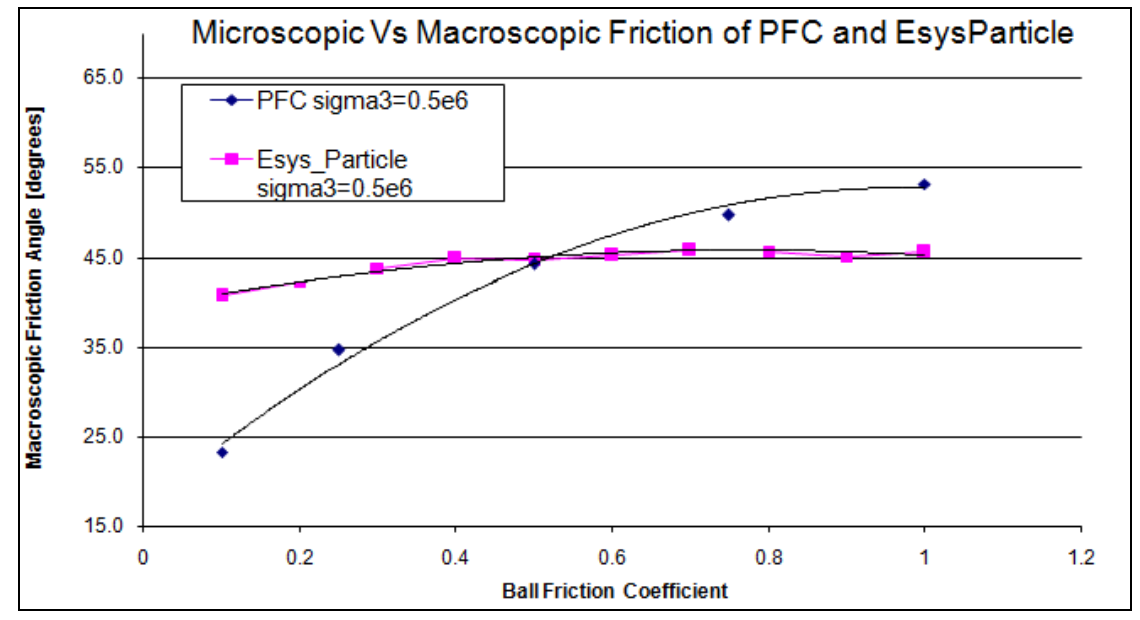

Figure 4 Macroscopic friction angle using a packing similar to PFC3D

Using a broader size distribution of particles ( 0.1 to $0.8 \mathrm{~m}$ radii) with lower porosity shows a slightly better response (see Figure 5) demonstrating the influence that particle packing has on macroscopic friction, with the final friction angle being seven degrees higher than previous tests.

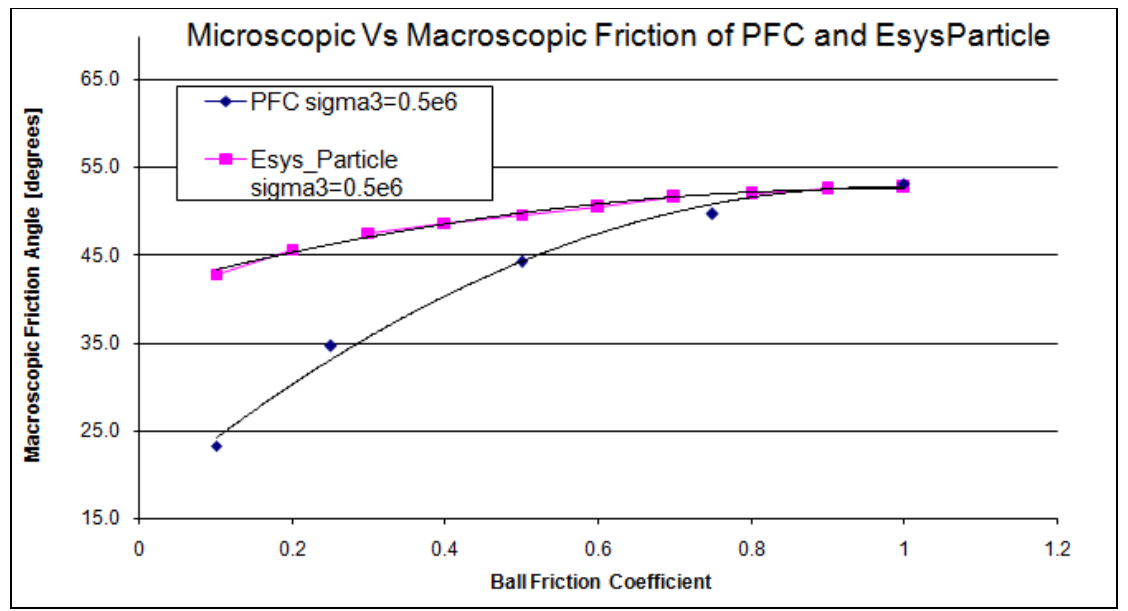

Figure 5 Macroscopic friction angle using a larger distribution packing 0.1 to $0.8 \mathrm{~m}$ Radii

A range of tests was also done with particle packing that resembles the packing currently used in the flow simulation tests, described in the following section. The parameters for these tests are shown in Table 2 . 
Table 2 Flow simulation particle properties

\begin{tabular}{ll}
\hline Parameter & Value \\
\hline Mean radius & $0.38 \mathrm{~m}(0.3-0.6 \mathrm{~m}$ range $)$ \\
Particle density & $3333 \mathrm{~kg} / \mathrm{m}^{3}$ \\
Particle stiffness & $0.1 \mathrm{GPa} \times \mathrm{R}_{\text {mean }}$ \\
Particle normal/particle shear stiffness & 1.0 \\
Particle static friction coefficient $\mu$ & $0.1-1.0$ \\
Particle dynamic friction coefficient $\mu$ & $0.1-1.0$ \\
Viscosity & 0.1 \\
\hline
\end{tabular}

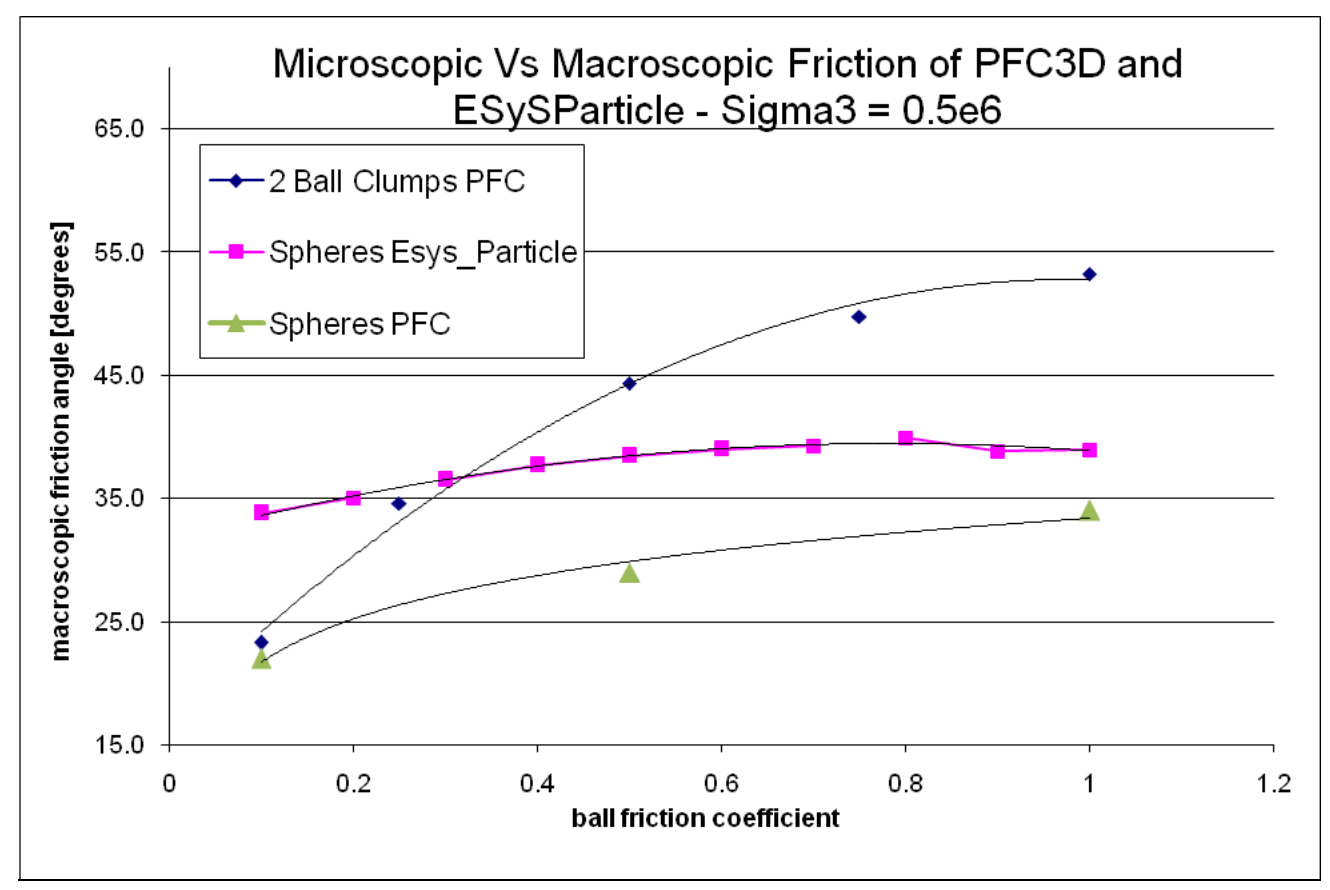

Figure 6 Macroscopic friction angle using quarter symmetry simulation parameters

The friction angle for the majority of testing being conducted on the quarter symmetry models is thought to be 38 degrees; this is seven degrees lower than the PFC3D clumps, however it is eight degrees higher than PFC3D spheres. Testing results on quarter symmetry presented later shows that, despite a lower friction angle, flow width results are similar for the model heights compared.

\subsection{D quarter symmetry flow model}

A quarter symmetry model has been constructed for numerical calibration against a previous flow study. In that study a quarter symmetry model was shown to have similar flow properties to a full symmetry model and, for computational reasons, the quarter symmetry model was selected as the test case. A number of ESyS-Particle quarter symmetry models were run using various particle packings and boundary conditions. The final result is a model which compares well with the results from PFC3D quarter symmetry tests. 


\subsubsection{Test setup description}

The layout used is $23 \times 18 \mathrm{~m}$ (length $\times$ width) with a quarter draw bell situated in the corner of the model measuring $2.5 \mathrm{~m}$ wide and $5 \mathrm{~m}$ deep. The drawbell uses a minor apex angle of approximately 60 degrees, a major angle of approximately 80 degrees and a draw bell height of $13.26 \mathrm{~m}$ high (from the top of the draw point), extended into the draw bell measuring $10.5 \mathrm{~m}$ wide and $7.5 \mathrm{~m}$ deep (PFC3D $7 \mathrm{~m}$ circular draw bell) at the top. The sidewalls and base walls contain a layer of fixed particles to produce a rough frictional wall and the draw bell and symmetry planes use frictionless walls.

\section{Table 3 Quarter symmetry model parameters}

\begin{tabular}{ll}
\hline Parameter & Value \\
\hline Mean radius & $0.38 \mathrm{~m}(0.3-0.6 \mathrm{~m}$ range $)$ \\
Particle density & $3333 \mathrm{~kg} / \mathrm{m}^{3}$ \\
Particle stiffness & $0.1 \mathrm{GPa} \times \mathrm{R}_{\text {mean }}$ \\
Particle normal/particle shear stiffness & 1.0 \\
Particle static friction coefficient $\mu$ & 0.5 \\
Particle dynamic friction coefficient $\mu$ & 0.5 \\
Viscosity & 0.01 \\
Packing height & $40,60,80,100,120 \mathrm{~m}$ \\
Particles & $29 \mathrm{~K}, 44 \mathrm{~K}, 58 \mathrm{~K}, 73 \mathrm{~K}, 87 \mathrm{~K}$ \\
\hline
\end{tabular}

The simulation is first packed into a settled state, which is usually one fifth of the total runtime. Once in its settled state a trapdoor, representing the bottom of the draw bell, is removed and the particles are allowed to flow out. Two main areas of flow are examined, that of a region of extracted particles which have exited the system and that of particles that have moved during the course of the flow. These regions allow calibration against previous studies.

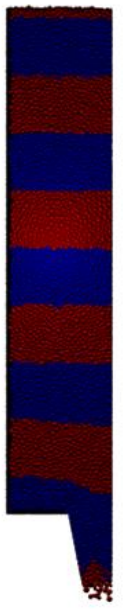

Figure 7 Side view of quarter symmetry model Figure 8 (100 $\mathrm{m}$ from base of model)

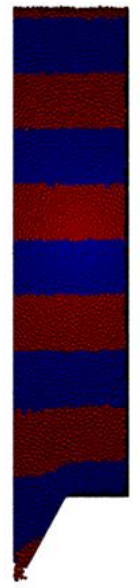

Front view of quarter symmetry model (100 $\mathrm{m}$ from base of model) 


\subsubsection{Quarter symmetry results}

In terms of gravity flow of rock, ESyS-Particle produces results similar to previous physical and numerical modelling studies in that flow shapes exhibit an elliptical characteristic. It can also be shown visually that the range of behaviours exhibited by the models after the settling phase is in line with that of granular flow, which is a positive sign with regards to the choice of parameters. A comparison between the movement zone region from ESyS-Particle (side plane) and PFC3D are shown in Figure 9 with good equivalence despite the differences in the friction angles of the particles, as discussed earlier.

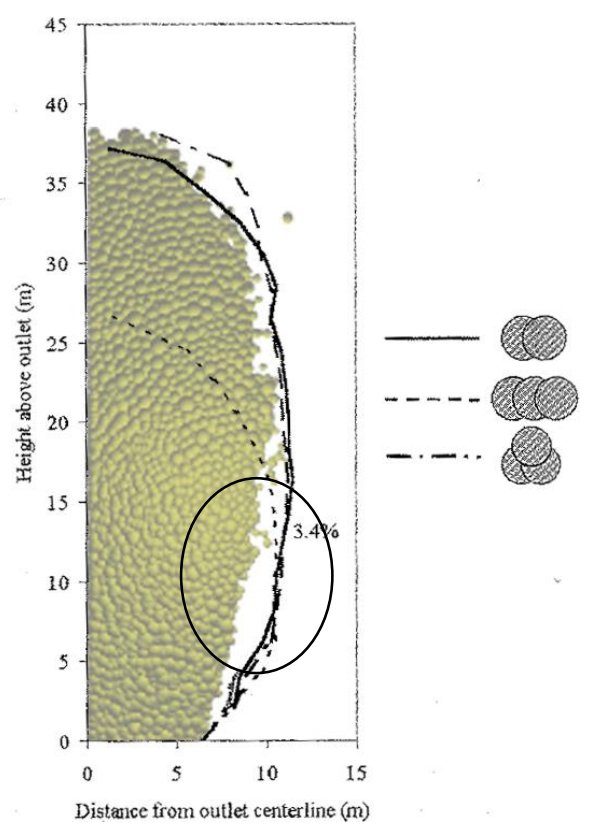

Figure 9 PFC3D (lines) to ESyS-Particle (particles) movement zone comparison

A major difference between both models currently, is in the modelling of the draw bell. The PFC3D model applies a velocity profile to particles exiting the system through the top of the draw bell, whereas in the ESyS-Particle model, particles flow through the draw bell and exit from the bottom of the draw bell. This difference manifests as a narrowing of the movement zone as compared with PFC3D, above the apex of the draw bell. These 3D simulations demonstrate that ESyS-Particle produces results that are not dissimilar from previous numerical models, providing confidence that ESyS-Particle is a suitable tool for flow modelling research.

\section{Conclusions and future directions}

Triaxial testing has uncovered some of the differences between ESyS-Particle and PFC3D and also offers some good insight into tackling the problem of calibration of ESyS-Particle with respect to block caving. The test results also provide explanation for the observed insensitivity of extraction zone widths to changes in the friction coefficient in the 2D flow simulations performed previously. Finally, these tests demonstrate the importance of the particle packing to achieve desired macroscopic material properties.

Further triaxial testing of particle packing used in the quarter symmetry runs has allowed for a better understanding of the relations between particle properties and flow widths. Through careful selection of properties and boundary conditions, ESyS-Particle has been able to be calibrated against a previous modelling study, and this result can be used as a demonstrator that both DEM codes, although with slightly different implementations, can be configured to mimic each other.

Future work is expected to revolve around extending the boundaries of current DEM flow modelling by using larger simulations and different flow situations. These problems include further particle size distribution testing, fines migration, the effects of controlled draw and compaction and interacting draw 
zones using any of the above. It is expected that this work will be able to complement previously written literature in the field and provide grounds for further modelling studies.

\section{Acknowledgements}

The author is grateful for the support of Rio Tinto for the funding of this work and for the co-operation and comments of M. Pierce, G. van Hout, A. van As and G. Chitombo. The help and experience of Y. Wang is also gratefully appreciated with regards to discussions about model setups and examination of results. Thanks also to F. Alonso-Marroquin for suggestions in preparation of this paper. The ESyS-Particle software was developed with funding support from the Australian Computational Earth Systems Simulator MNRF of AuScope Ltd. Numerical simulations were performed on the ESSCC SGI Altix 3700 supercomputer funded by the Queensland State Government and ACCESS MNRF.

\section{References}

Abe, S., Place, D. and Mora, P. (2004) A parallel implementation of the lattice solid model for the simulation of rock mechanics and earthquake dynamics. Pure Appl. Geophys, Vol. 161, pp. 2265-2277.

Castro, R. (2006) A study of isolated draw zones in block caving mines. Brisbane, University of Queensland.

Cundall, P. and Strack, O. (1979) A discrete numerical model for granular assemblies. Geotechnique, Vol. 29, pp. 47-65.

Julin, D. (1992) Block Caving. H. Hartman (editor) SME Mining Engineering Handbook. Littentlon, Society for Mining Metallurgy and Exploration, pp. 1815-1836.

Latham, S., Abe, S. and Davies, M. (2004) Scaling Evaluation of the Latticle Solid Model on the SGI Altix 3700. 7th International Conference on High Performance Computing and Grid in the Asia Pacific Region (HPCAsia2004), Tokyo, pp. 226-233.

Latham, S., Abe, S. and Mora, P. (2005) Macroscopic Friction Response of Rotational and Non-Rotational Lattice Solid Gouge Models in 2D and 3D. Powders and Grains Stuttgart, Germany.

Lorig, L. and Cundall, P. (2000) A rapid gravity flow simulator, Final Report, International Caving Study, E.T. Brown (editor), Brisbane, JKMRC and Itasca Consulting Group, Inc.

Mora, P. and Place, D. (1994) Simulation of the stick-slip instability. Pure Appl. Geophys, Vol. 143, pp. 61-87.

Pierce, M., Cundall, P., Van Hout, G. and Lorig, L. (2003) PFC3D Modeling of Caved Rock Under Draw. Numerical Modeling in Micromechanics via Particle Methods, H. Konietzky (editor), Proceedings 1st International PFC Symposium, November 2002, Gelsenkirchen, Germany, Balkema.

Power, G. (2004) Modelling Granular Flow in Caving Mines: Large Scale Physical Models and Full Scale Experiments. Brisbane, University of Queensland.

Wang, Y., Abe, S., Latham, S. and Mora, P. (2006) Implementation of Particle-scale Rotation in the 3-D Lattice Solid Model. Pure and Applied Geophysics, Vol. 163, pp. 1769-1785.

Wang, Y. and Mora, P. (2007) A new algorithm to model particle rotation and parameter calibration in a 3D bonded DEM model. International Conference of Discrete Element Methods. Brisbane. 
